\title{
CSI in Supernova Remnants
}

\author{
You-Hua Chu \\ Institute of Astronomy and Astrophysics, Academia Sinica \\ email: yhchu@asiaa.sinica.edu.tw
}

\begin{abstract}
Supernovae (SNe) explode in environments that have been significantly modified by the SN progenitors. For core-collapse SNe, the massive progenitors ionize the ambient interstellar medium (ISM) via UV radiation and sweep the ambient ISM via fast stellar winds during the main sequence phase, replenish the surroundings with stellar material via slow winds during the luminous blue variable (LBV) or red supergiant (RSG) phase, and sweep up the circumstellar medium (CSM) via fast winds during the Wolf-Rayet (WR) phase. If a massive progenitor was in a close binary system, the binary interaction could have caused mass ejection in certain preferred directions, such as the orbital plane, and even bipolar outflow/jet. As a massive star finally explodes, the SN ejecta interacts first with the CSM that was ejected and shaped by the star itself. As the newly formed supernova remnant (SNR) expands further, it encounters interstellar structures that were shaped by the progenitor from earlier times. Therefore, the structure and evolution of a SNR is largely dependent on the initial mass and close binarity of the SN progenitor. The Large Magellanic Cloud (LMC) has an excellent sample of over 50 confirmed SNRs that are well resolved by Hubble Space Telescope, Chandra X-ray Observatory, and Spitzer Space Telescope. These multi-wavelength observations allow us to conduct stellar forensics in SNRs and understand the wide variety of morphologies and physical properties of SNRs observed.
\end{abstract}

Keywords. supernovae: general, supernova remnants, circumstellar matter, Magellanic Clouds

\section{Introduction}

There are two broad origins of supernovae (SNe): core-collapse SNe (CCSNe) originating from massive stars at the end of their evolution, and Type Ia SNe (SNe Ia) originating from white dwarfs in close binary systems. In the case of CCSN, the massive star progenitor had interacted with the ambient medium via UV ionising radiation and stellar winds before the star exploded as SN; thus the SN ejecta interacts with a surrounding that had been shaped and conditioned by the progenitor. The physical structure of the resulting supernova remnant (SNR) inevitably reveal imprints of the massive star progenitor, hence the study of CC SNRs may shed light on the nature of the massive progenitors.

SNe Ia are known to involve white dwarfs, but it is not known whether one or two white dwarfs are involved. The former, called single-degenerate (SD), requires a white dwarf accreting material from a close stellar companion whose envelope has expanded past the Roche limit (Whelan \& Iben 1973; Nomoto 1982). The stellar companion is heated/shocked by the impact of SN ejecta, but survives the explosion. The latter, called double-degenerate (DD), is produced by the merger of two white dwarfs (Iben \& Tutukov 1984; Webbink 1984), and after the explosion neither white dwarf remain. Therefore, if a surviving stellar companion is identified in a Type Ia SNR, the SD origin of the $\mathrm{SN}$ progenitor can be affirmed. The SD progenitor may produce a dense circumstellar medium (CSM) due to the mass loss of the stellar companion; therefore, a dense CSM detected in a Type Ia SNR also suggests a SD origin of the SN progenitor. 
SNRs, particularly the young ones, provide sites where we can carry out CSI (circumstellar investigation, or crime scene investigation) to assess the nature of the SN progenitors. In this paper, I review how massive stars modify their surrounding medium and how this process affects the physical structures of their SNRs. I will use CCSNRs in the Large Magellanic Cloud (LMC) to demonstrate the imprints of their massive progenitors. I will also use Type Ia SNRs in the LMC to search for surviving companions and CSM for evidence of SD SNe Ia.

\section{CSI in Core-Collapse SNRs}

Massive stars are sources of UV radiation that can photoionize the ambient medium. They are also sources of stellar winds of velocities that vary along the stellar evolution. When a massive star is on the main sequence, it is surrounded by the interstellar medium (ISM). A main sequence $O$ star has fast stellar winds with velocities of $1000-2000 \mathrm{~km}$ $\mathrm{s}^{-1}$ and mass loss rates of order of $10^{-7} M_{\odot} \mathrm{yr}^{-1}$. The star not only photoionizes the ambient ISM into an HII region, but also sweeps up the ISM into a wind-blown bubble (Castor, McCray, \& Weaver 1975; Weaver et al. 1977). As a massive star evolves off the main sequence to become a red supergiant (RSG) or a luminous blue variable (LBV), the fast stellar wind is replaced by a slow wind with velocity of $10-50 \mathrm{~km} \mathrm{~s}^{-1}$ and a mass loss rate of order of $10^{-4} M_{\odot} \mathrm{yr}^{-1}$. This slow wind expands away from the central star to form a CSM within the central cavity of the interstellar bubble previously blown by the main sequence progenitor. As the massive star evolves further into the Wolf-Rayet (WR) phase, the slow wind is replaced by a copious fast wind of velocities $2000-3000 \mathrm{~km}$ $\mathrm{s}^{-1}$ and mass loss rate of $\sim 10^{-5} M_{\odot} \mathrm{yr}^{-1}$. This fast wind sweeps up the CSM to form a circumstellar bubble.

It may have been puzzling that no interstellar bubbles around main sequence $\mathrm{O}$ stars as spectacular as the famous Bubble Nebula have been detected, especially since an O star's fast stellar wind should blow a bubble in the ISM. To definitively search for interstellar bubbles around main sequence O stars, Hubble Space Telescope (HST) images were obtained for the young HII regions N11B and N180B around OB associations LH10 and LH117, respectively; still, no bubbles can be identified morphologically in the $\mathrm{H} \alpha$ or $[\mathrm{O}$ III] images. The breakthrough came from the long-slit high-dispersion echelle spectra of these HII regions. Expanding shell structures around $\mathrm{O}$ stars are detected in these spectrograms; the shell sizes are $\sim 15 \mathrm{pc}$ and the expansion velocities are $15-20 \mathrm{~km}$ $\mathrm{s}^{-1}$ (Nazé, Chu, Points, et al. 2001). It is conceivable that these expansion velocities produce shocks with a Mach number of $1.5-2$, and such weak shocks do not cause strong compression to form dense interstellar shells that are identifiable morphologically.

The visibility of an interstellar bubble of an $\mathrm{O}$ star depends on the existence of other massive stars in its vicinity and the distribution of the ISM. For example, the interstellar bubble in the Orion Nebula is not clearly identified until XMM-Newton detected diffuse $\mathrm{X}$-ray from the bubble interior, then it became obvious that the massive stars are close to the densest parts of the bubble and the less dense parts of the bubble extend further away from the stars (Güdel, Briggs, Montmerle, et al. 2008). Massive stars in OB associations or clusters will collectively form superbubbles with sizes larger than those of single-star interstellar bubbles. In an active star-forming region, such as the giant HII region 30 Doradus, the stellar energy feedback produce very complex structures, and effects of individual stars cannot be unambiguously identified.

The ambient medium of a massive star can be roughly characterized by the following physical parameters: interstellar bubble around a main sequence $\mathrm{O}$ star has density of 10-100 H-atom $\mathrm{cm}^{-3}$, diameter of a few 10's pc, and expansion velocity $\sim 15 \mathrm{~km} \mathrm{~s}^{-1}$; 

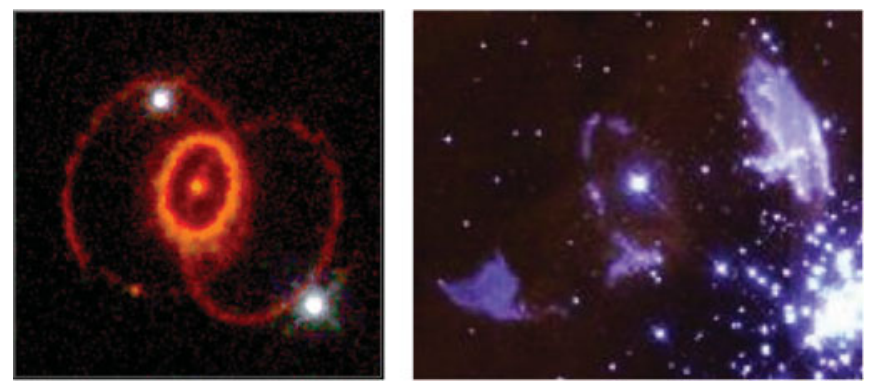

Figure 1. Left - Hubble Space Telescope image of SN1987A. Right - Hubble Space Telescope image of Sher 25.
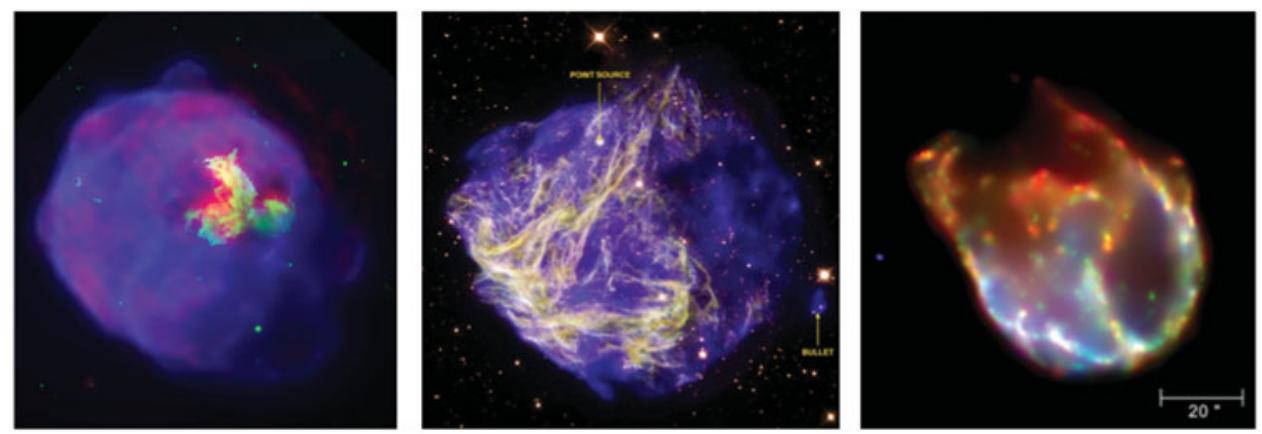

Figure 2. Left to right - Hubble Space Telescope images of N63A, N49, and Chandra X-ray image of N132D.

circumstellar nebula around a RSG has $>10^{4} \mathrm{H}$-atom $\mathrm{cm}^{-3}, 10^{3} \mathrm{AU}$, and $10-50 \mathrm{~km}$ $\mathrm{s}^{-1}$; circumstellar nebula around an LBV has $10^{4} \mathrm{H}$-atom $\mathrm{cm}^{-3}, 1 \mathrm{pc}$, and 20-50 km $\mathrm{s}^{-1}$; circumstellar bubble around a WR star has $10^{2} \mathrm{H}$-atom $\mathrm{cm}^{-3}$, a few pc, and 50 $100 \mathrm{~km} \mathrm{~s}^{-1}$. The detailed physical conditions of the ambient medium at the time of SN explosion depend on the exact evolutionary path and mass loss history. When a massive star explodes at the end of its life, the SN ejecta interacts successively with different layers of ambient medium, first CSM then ISM. The different conditions of the ambient medium produce a wide variety of physical structures in SNRs. Below I will use SNRs in the LMC to illustrate interactions between SN ejecta and the ambient medium.

SN1987A provides a clear example of SN ejecta interacting with a CSM (Figure 1). The progenitor of SN1987A, Sk-69 201, was a B3 I supergiant (Walborn, Lasker, Laidler, et al. 1988). The structure of Sk-69 202's CSM is almost identical to the CSM of Sher 25, a Galactic B1.5 I supergiant (Brandner, Chu, Eisenhauer, et al. 1997). Interestingly, the second youngest CCSNR in the LMC, 0540-69.3, also shows a [N II] ring around the SN that corresponds to a CSM similar to that around Sk-69 202 or Sher 25 (Caraveo, Mignani, \& Bignami 1998; Sandin, Lundqvist, Lundqvist, et al. 2013).

As massive stars had blown interstellar bubbles during the main sequence phase, their SN ejecta will quickly sweep up the CSM and expand into the cavity of the bubble. Indeed, many young CCSNRs show indications of cavity explosion, for example N63A, N49, and N132D in the LMC (Figure 2). As noted earlier, interstellar bubbles of main sequence O stars do not show strong compression in the shell, so such bubbles are better described as interstellar cavities. When SN ejecta expands into the cavity, the expansion velocity is not dampened by dense material, so the X-ray image can show high luminosities in 

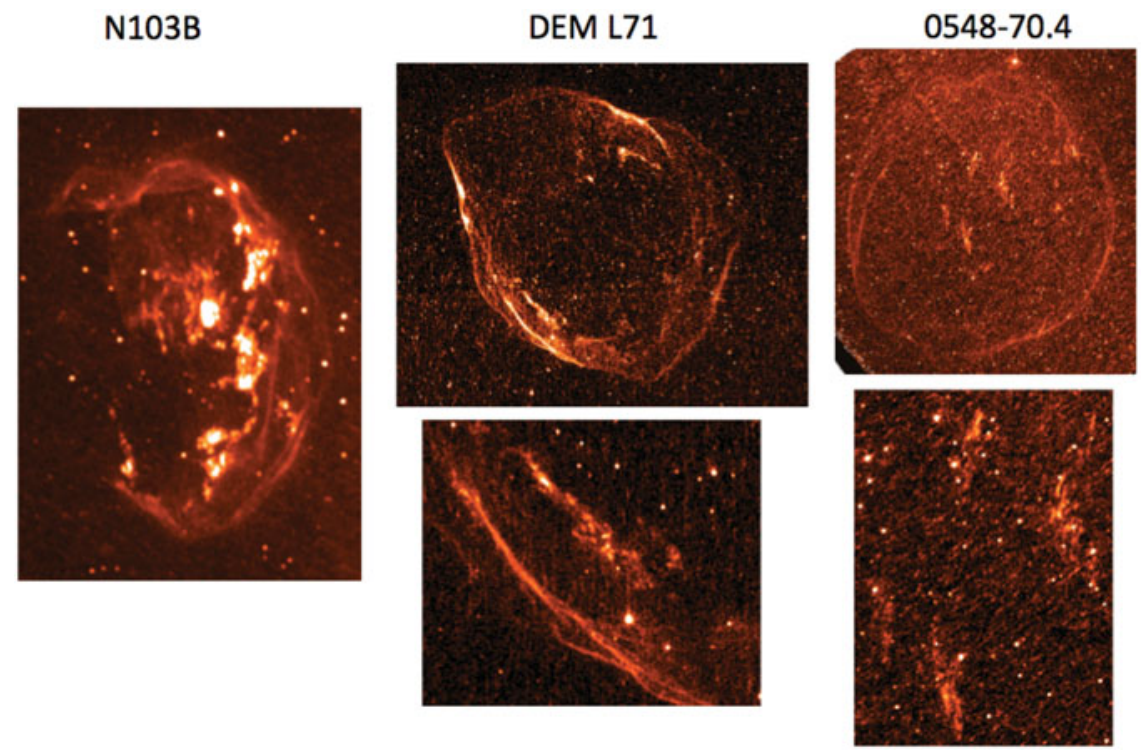

Figure 3. Hubble Space Telescope images of N103B, DEM L71, and 0548-70.4. The lower panels show close-ups of the knots.

CCSNRs that are already $>20$ pc in size (Warren, Hughes, Slane, et al. 2003; Park, Hughes, Slane, et al. 2012; Vogt \& Dopita 2011).

\section{CSI in Type Ia SNRs}

Type Ia SNRs can be diagnosed by the over-abundance of Fe, Si, S, Ar, and Ca implied by the X-ray spectra of the SN ejecta (e.g., Hughes, Hayashi, Helfand, et al. 1995). Young Type Ia SNRs can also be diagnosed by their Balmer-dominated optical spectra due to collisionless shocks into a mostly neutral ISM (Chevalier, Kirshner, \& Raymond 1980). They can also be unambiguously identified by spectra of their light echoes (Rest, Suntzeff, Olsen, et al. 2002; Rest, Matheson, Blondin, et al. 2005).

If CSM is detected in a Type Ia SNR, the SN progenitor is most likely of SD origin. Hubble Space Telescope images of Type Ia SNRs in the LMC have revealed dense knots in the interior of N103B, DEM L71, and 0548-70.4. These knots have densities a few thousand H-atoms $\mathrm{cm}^{-3}$, which is more characteristic for CSM than ISM.

The Hubble Space Telescope H $\alpha$ image of N103B shows a filamentary shell with dense knots in the shell interior *(Figure 3). High-dispersion long-slit echelle spectra of N103B show that the filamentary shell is Balmer-dominated while the dense knots show forbidden lines (Figure 4), clearly indicating that the knots belong to a CSM (Li, Chu, Gruendl, et al. 2017).

The dense CSM in N103B suggests that its SN progenitor has a SD origin. It is thus interesting to search for a surviving companion of the progenitor. A candidate has been identified near the explosion center defined by the Balmer-dominated shell ( $\mathrm{Li}$, Chu, Gruendl, et al. 2017). High-quality spectra of this star is needed to examine its radial velcoity, rotation velocity, and abundances in order to confirm its identification as the surviving companion.

This work was supported by the grant 104-2112-M001-044MY3 from the Ministry of Science and Technology of Taiwan. 

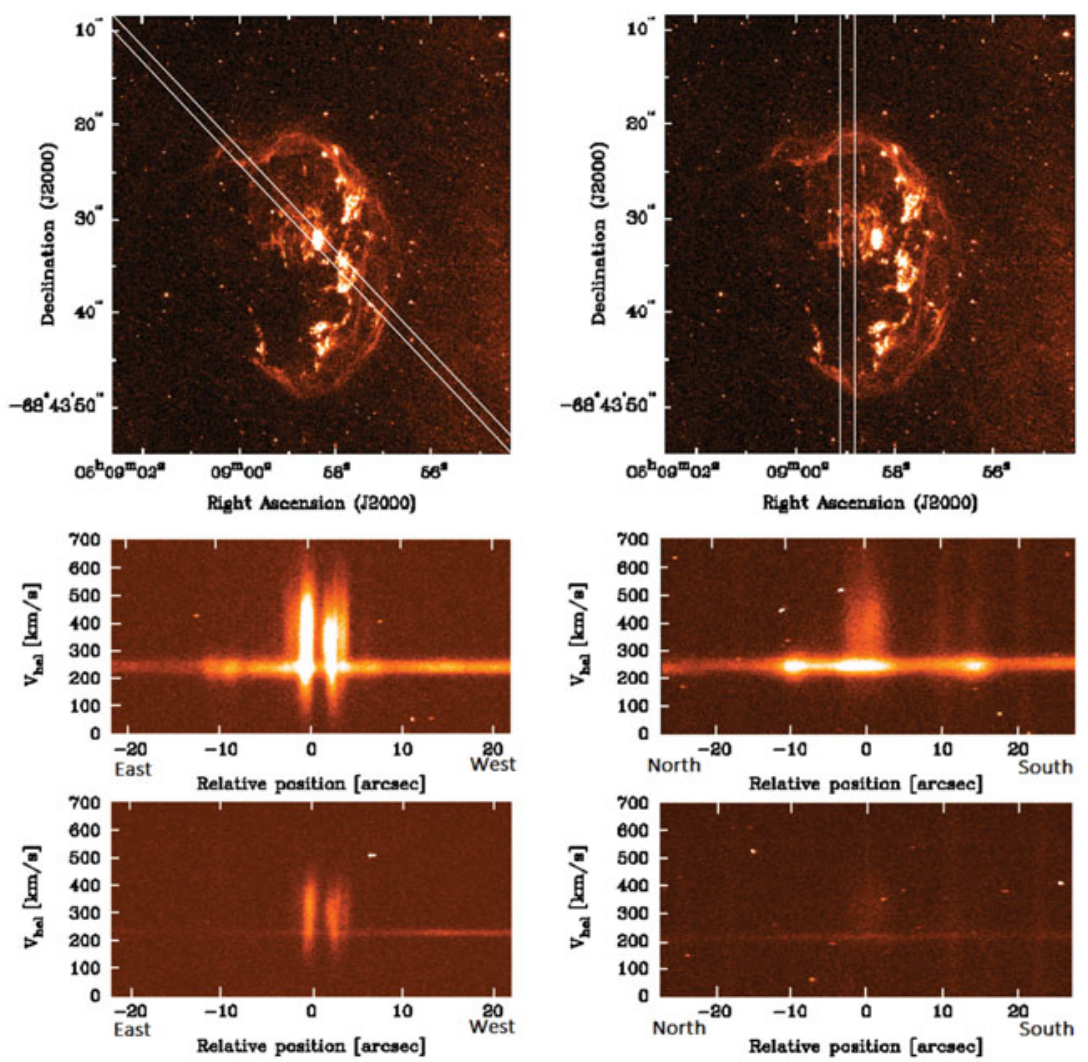

Figure 4. Top panel: Hubble Space Telescope H $\alpha$ image of N103B. Bottom panels: long-slit echelle spectrograms of the $\mathrm{H} \alpha$ and $[\mathrm{N} \mathrm{II}] \lambda 6583$ lines. It is clear that the filamentary shell has only $\mathrm{H} \alpha$ emission and no [N II], while the knots are shocked dense CSM with bright [N II] emission along with the $\mathrm{H} \alpha$.

\section{References}

Brandner, W., Chu, Y.-H., Eisenhauer, F., Grebel, E. K., \& Points, S. D. 1997, ApJL, 489, L153

Caraveo, P., Mignani, R., \& Bignami, G. B. 1998, Mem. Soc. Astronomia Italiana, 69, 1061

Castor, J., McCray, R., \& Weaver, R. 1975, ApJL, 200, L107

Chevalier, R. A., Kirshner, R. P., \& Raymond, J. C. 1980, ApJ, 235, 186

Güdel, M., Briggs, K. R., Montmerle, T., et al. 2008, Science, 319, 309

Hughes, J. P., Hayashi, I., Helfand, D., et al. 1995, ApJL, 444, L81

Iben, I., Jr. \& Tutukov, A. V. 1984, ApJS, 54, 335

Li, C.-J., Chu, Y.-H., Gruendl, R. A., et al. 2017, ApJ, 836, 85

Nazé, Y., Chu, Y.-H., Points, S. D., et al. 2001, AJ, 122, 921

Nomoto, K. 1982, ApJ, 257, 780

Park, S., Hughes, J. P., Slane, P. O., et al. 2012, ApJ, 748, 117

Rest, A., Matheson, T., Blondin, S., et al. 2008, ApJ, 680, 1137

Rest, A., Suntzeff, N. B., Olsen, K., et al. 2005, Nature, 438, 1132

Sandin, C., Lundqvist, P., Lundqvist, N., et al. 2013, MNRAS, 432, 2854

Vogt, F. \& Dopita, M. A. 2011, Ap. Es Space Sci., 331, 521

Walborn, N. R., Lasker, B. M., Laidler, V. G., \& Chu, Y.-H. 1987, ApJL, 321, L41

Warren, J. S., Hughes, J. P., \& Slane, P. O. 2003, ApJ, 583, 260

Weaver, R., McCray, R., Castor, J., Shapiro, P., \& Moore, R.1977, ApJ, 218, 377

Webbink, R. F. 1984, ApJ, 277, 355

Whelan, J. \& Iben, I., Jr. 1973, ApJ, 186, 1007 